\title{
LA ABSORCIÓN DE FUERZA DE TRABAJO EN EL SECTOR INDUSTRIAL
}

\author{
GustaV RANis * \\ Economic Growth Center \\ Universidad Yale
}

EL INTERÉS reciente y cada vez mayor por el problema del desempleo en los países en vía de desarrollo (PVD) adquiere muchas formas. Al igual que el desarrollo mismo, el tema es complejo y presenta muchas facetas, pero es posible que ninguno de los eslabones en la cadena de argumentos haya sido tan incierto y controvertido como el referente a la gama real de opciones tecnológicas abiertas físicamente a los sectores no agrícolas de los PVD de hoy en día - aun cuando hubiera disposición a escuchar consejos pertinentes sobre el tema.

En este trabajo se intenta arrojar luz sobre la cuestión de la capacidad de los sectores industriales de los PVD para absorber eficientemente la mano de obra desempleada o subempleada en el curso del desarrollo económico. Sabemos que inclusive en los países que han crecido a una tasa de 5 ó $6 \%$ anual en términos reales en el pasado -y cuyo crecimiento global ha sido en general muy satisfactorio en los años sesenta, tal como lo registra la Comisión Pearson- las tasas de crecimiento del sector industrial de 8 a $10 \%$ anual han estado acompañadas por tasas de absorción de fuerza de trabajo de solamente 2 ó 3\%. Por añadidura, la elasticidad del empleo industrial con respecto a la producción no solamente ha sido baja, sino que al parecer ha disminuido a lo largo del tiempo. ${ }^{1}$ En consecuencia, en casi todo el mundo subdesarrollado parece que los índices combinados de desempleo y subempleo han estado creciendo - acompañados de una conciencia cada vez mayor de lo inadecuado del ingreso per capita como indicador principal del proceso.

Las dimensiones del problema se aprecian si este sombrío panorama histórico se combina con el hecho de que aun cuando se adoptaran de inmediato en todas partes políticas de "crecimiento nulo de la población", la actual estructura por edades de la población de los países en desarrollo resultará en una explosión de la fuerza de trabajo de grandes proporciones (aumento de 3.5 a $4 \%$ anual en el próximo decenio). Si

* El autor fue profesor visitante del Centro de Estudios Económicos de El Colegio de México de septiembre de 1971 a junio de 1972; el presente artículo fue publicado como "Center Discussion Paper No 116" y forma parte de un trabajo elaborado para el Banco Mundial.

1 Véase Werner Baer y Michel E. A. Hervé, "Employment and Industrialization in Developing Economies", Quarterly Journal of Economics, vol. LXXX, núm. 1, febrero de 1966. 
el mundo en desarrollo fuera incapaz de absorber estos aumentos avisorados de la fuerza de trabajo - aparte de eliminar el monto sustancial de subempleo ya existente en la mayoría de los países- las perspectivas son ciertamente sombrías.

El papel del sector industrial en este contexto - aun cuando definamos éste en términos amplios que incluyan todo lo no agrícola salvo los servicios no productivos - atendería desde luego a sólo una parte del problema. Si un PVD se desarrolla, es probable que en el proceso tenga que activar su sector agrícola preponderante. El que en este contexto el aumento de la productividad agrícola economice o no fuerza de trabajo tiene gran importancia en relación con el tamaño de la carga que recaerá sobre la absorción de trabajo por el sector no agrícola, cualquiera que fuere la tasa de crecimiento global. Pero en este trabajo el interés se centrará en la capacidad de los sectores no agrícolas para absorber empleo en forma efíciente. No sólo existe incomprensión al respecto -por alguna razón la mayoría de la gente parece aceptar el realismo de escalas, tenencia y combinaciones de insumos agrícolas alternativos, mientras que sostienen religiosamente las proporciones fijas en la industria - sino que sabemos también, independientemente del resultado de las investigaciones acerca de las consecuencias de la revolución verde sobre el empleo, que con el tiempo la gente inevitablemente será desplazada de la agricultura, en el caso de que haya éxito, o será extraída de ella, en el caso de un fracaso - y que la capacidad del sector no agrícola para absorberlos de manera eficiente jugará un papel decisivo en cualquier versión global equilibrada del desarrollo.

En la sección II se intenta colocar el panorama generalmente desfavorable de la absorción de trabajo industrial en su perspectiva histórica y política adecuada. En la sección III se adelantan algunas nociones preliminares sobre la naturaleza diferencial del proceso innovativo a través del tiempo en tal contexto, y en la sección IV se procura demostrar la importancia empírica de estas nociones, en condiciones de ambiente político favorable, haciendo referencia al Japón histórico y a Corea y Taiwán contemporáneos.

\section{II}

Ya ha pasado a formar parte de la sabiduría convencional la crítica a la llamada sustitución de importaciones adoptada por la mayoría de los PVD durante los años cincuenta y sesenta. Este régimen comprendió por lo regular el conjunto de políticas que incluían controles de divisas y permisos de importación, déficit presupuestales e inflación y tasas reales de interés bajas (a veces negativas). La meta, hablando en términos generales, era la de redirigir los flujos colonialistas tradicionales - preindependientes - hacia la creación de infraestructura social y económica y el reemplazo de las importaciones por industrias productivas de bienes de consumo. La consecuencia sobre el funcionamiento de la economía fue con frecuencia un crecimiento súbito, pero ineficiente, de la producción industrial, es decir, intensivo en el uso de capital y de importaciones, acompañado por un desestímulo de las exportaciones y de la producción agrícola, tasas reducidas de empleo industrial, de cambio tec- 
nológico y de ahorro interno, y una dependencia relativamente fuerte de ayuda externa.

A medida que los gobiernos de los PVD se fueron dando cuenta de los costos económicos de este conjunto de políticas, durante los años sesenta pudo observarse una tendencia, al menos en algunos países, a considerar un conjunto alternativo de políticas. Este conjunto puede describirse, aunque una vez más tengamos que sobresimplificar, como algo que apunta a la reducción de algunas de las ineficiencias más burdas que aquejan al desarrollo industrial a través del reajuste de diversos precios relativos esenciales que previamente fueron deformados, incluidas las tasas de interés y los tipos de cambio. Al reemplazar los controles cuantitativos del mercado de divisas por aranceles y avanzar hacia tipos de cambio más realistas, a través ya sea de una devaluación de hecho o de derecho, y sustituir el racionamiento severo del crédito con tasas de interés más altas, el acceso al desarrollo y la participación pudieron por primera vez ofrecerse a los empresarios medianos y pequeños. En el curso de esta segunda fase, o sea la de sustitución de exportaciones, el desarrollo industrial se basa menos en los recursos naturales y más en los recursos humanos; ya no se discrimina en contra de las exportaciones -en especial las no tradicionales e intensivas de trabajo-, las tasas de ahorro interno pueden empezar a aumentar y se va hacia la etapa de despegue de que habla Rostow; la agricultura ya no queda perjudicada por la relación desfavorable de precios del intercambio y puede jugar su papel histórico en la generación de excedentes, los cuales, debidamente canaiizados, pueden dar simultáneamente empleo a la fuerza de trabajo no calificada que a la vez es liberada; y el cambio tecnológico local en ambos sectores puede asumir una importancia mayor.

Aunque en el mundo subdesarrollado no existe aún una tendencia notable hacia tal política de sustitución de exportaciones, su adopción ciertamente ha tenido resultados importantes, como sucede por ejemplo en Corea, Taiwán y Paquistán occidental, al convertir algunas situaciones de estancamiento virtualmente general, como en los años cincuenta, en una de crecimiento elevado en los sesenta.

Tal vez, para el argumento presente, lo más importante sea el hecho de que las nuevas y mejores señales de esta fase probablemente induzcan la adopción de tecnologías y combinaciones de productos que hagan uso más intensivo de la mano de obra o reduzcan el desempleo. En este contexto, para bien o para mal, los flujos tecnológicos entre los países ricos y los países pobres jugarán inevitablemente un papel vital. La mera coexistencia de países a niveles tecnológicos muy diferentes tiene que representar una de las influencias más importantes en el funcionamiento pasado, actual y futuro de los PVD. Es la naturaleza precisa de estos flujos tecnológicos y la manera en que los PVD los han absorbido lo que, en nuestra opinión, ha tenido un impacto decisivo en el funcionamiento global durante los dos últimos decenios de desarrollo. En otras palabras, es también en esta área donde puede y debe localizarse el mayor potencial para mejorar el funcionamiento de los PVD durante los años setenta.

Bajo la influencia de la historia pasada muchos han llegado a la conclusión de que existe un conflicto inevitable entre los dos objetivos de empleo y crecimiento. Pero antes de aceptar tales conclusiones fundamentalmente negativas, estamos obligados a examinar con cuidado la 
validez de la proposición, en especial en países que parecen haber funcionado bien en el pasado con respecto a la producción y al empleo. Ciertamente, tal examen es necesario antes de que podamos de modo inteligente dirigirnos a la cuestión de cómo el mundo en desarrollo en su totalidad podrá absorber de manera eficiente los aumentos inevitables de su fuerza de trabajo, para no hablar de absorber la carga ya existente de desempleados y subempleados.

En la perspectiva de los años setenta parecen estar surgiendo tres escuelas de pensamiento con respecto a la solución del problema del desempleo. Una sugiere que es necesario crecer más de prisa, es decir, a una tasa de crecimiento mayor a la que se alcanza tradicionalmente, con "filtración" suficiente para alcanzar el pleno empleo. Ésta es claramente, en el mejor de los casos, una proposición cara y poco realista, que requiere de enormes volúmenes de capital extranjero, por ejemplo, si la débil maquinaria dominada por la sustitución de importaciones de los años sesenta ha de rendir, sin reestructuración, sustancialmente más empleo. Un segundo enfoque supone también que no haya cambio en los parámetros de las relaciones de conducta del sistema, pero indica que después de que el ejercicio de planeación tradicional haya sido consumado, se emplee una "estrategia suplementaria" para eliminar el desempleo restante. Esto de ordinario significa instituir programas de obras públicas que utilicen intensamente mano de obra, ya sea en las áreas rurales o en las urbanas. Existe aquí una posibilidad real, en especial cuando por ejemplo la infraestructura rural es inadecuada - pero la evidencia hasta ahora indica que la capacidad de planeación y ejecución pueden ser una restricción al pasar de un proyecto a otro. Pero lo que resulta más importante es que este enfoque no hace del problema del empleo parte de la estrategia primordial de desarrollo y lo relega a una mera adición, la cual - a pesar de las protestas de todos los planes quinquenales sobre lo contrario- fue la situación corriente durante los años cincuenta y sesenta.

El tercer enfoque intenta cambiar la naturaleza del patrón de crecimiento mismo haciéndolo más sensible a la dotación básica de factores. Esto significa que no intentamos "destronar al producto nacional bruto", salvo quizá en términos políticos, sino que intentamos colocarlo en un trono más firme. En otras palabras, una vez que la economía dual abierta se sale de su posición artificial de precios administrados y sustitución de importaciones, y se dirige a una fase más orientada hacia el mercado a través de sustitución de exportaciones, se hace posible que ocurran cambios de gran importancia en la combinación de productos y en la tecnología -en ambos casos hacia un mayor empleo de mano de obra. Tal reestructuración, tal como puede demostrarse en los casos de Taiwán y Corea, puede permitir a la economía tener más de ambas cosas, esto es, más producción y más empleo, en lugar de tener que elegir entre ellos. Más aún, tal movimiento hacia una orientación del mercado no tiene en lo absoluto nada que ver, como con frecuencia se alega, con un regreso al colonialismo o a entregar el país a la libre empresa. La misma conveniencia de dar beligerancia a la dotación de factores en las decisiones sobre la producción tiene vigencia en los países socialistas $\mathrm{y}$, de hecho, en nuestros días el mercado se usa cada vez más como instrumento de los objetivos de la planificación socialista de Europa oriental. 
Por último, debe hacerse notar que la distribución del ingreso, una tercera dimensión cada vez más importante para el funcionamiento del desarrollo, se ve también beneficiada. Hay quienes suponen que cualquier tendencia a mantener los salarios a nivel bajo, conforme a la condición de excedentes de fuerza de trabajo, debe ser mala para el "hombre pequeño". De hecho, la situación es más bien la contraria. Cuando el problema de la pobreza sea en parte considerable un problema de desempleo - debido ya sea a la falta de deseo o a la capacidad inadecuada del gobierno para efectuar una redistribución por vía fiscal- el "hombre pequeño" que es dañado por la restricción salarial es el que ya tiene empleo, no el que suele estar relegado, desempleado o subempleado. Probablemente no sólo la producción total, y de aquí el ingreso per capita, sino también la nómina total de salarios se elevarán una vez que la economía se dirija hacia tecnologías y combinaciones de producto que utilicen mano de obra intensivamente. Las cifras sobre distribución del ingreso de varios países indican, lo cual es muy interesante, que Taiwán es igualmente el país que mejor funciona en este sentido, esto es, el que observa en la muestra la mejor distribución del ingreso. Todavía más instructivo es el hecho de que Taiwán muestra ser el que mejor funciona a este respecto en 1964 - cuando se encontraba en auge la sustitución de importaciones- que en 1953, durante su propia fase de sustitución de importaciones. Aunque no lo admitan los economistas condicionados a pensar siempre en términos de beneficios alternativos (trade-offs), los países con abundancia de mano de obra, dentro de su frontera de eficiencia, pueden ser capaces de disfrutar simultáneamente de más empleo, mayor crecimiento y mejor distribución del ingreso, si se dan los cambios adecuados de política económica.

Al percatarse de esto, se dirige mucha más atención ahora a la importancia de los factores deformadores de los precios, a los tipos de cambio sobrevaluados, a las políticas fiscales inadecuadas, a la legislación sobre bienestar "prematura", y a otras restricciones institucionales que obviamente han conducido en parte a una tasa baja de utilización de la fuerza de trabajo, en especial en los crecientes sectores no agrícolas de los PVD. Pero mucha menos atención se ha dado hasta la fecha a las alternativas tecnológicas realmente disponibles para un PVD típico -en el supuesto de que sus empresarios y su gobierno lleguen a encarar el problema de adoptar un conjunto más confiable de señales relativas a la dotación de factores y a las calificaciones existentes. En otras palabras, aun cuando las políticas fiscales, monetarias y de crédito y de tasas de interés fueran ideales, ¿la elección de tecnología entre la disponible en el exterior o producible en el país sería sustancialmente distinta? ¿O existen otras limitaciones severas a la elección que obligan a los PVD a aceptar la tecnología corrientemente disponible en la mayoría de los países avanzados exportadores de capital?

Muchos funcionarios de los PVD, de los donantes de ayuda, y economistas académicos insisten todavía en que la mayor parte del cambio tecnológico, en especial el no agrícola, debe ocurrir en el exterior, y que el país prestatario subdesarrollado tiene ante sí de hecho solamente un conjunto muy estrecho de opciones tecnológicas. Si sólo son pertinentes los coeficientes correspondientes a la maquinaria más reciente producida en los países más avanzados, toda la discusión sobre proporciones 
alternativas de factores como respuesta a dotaciones alternativas de recursos, se vuelve en gran medida inútil, o se limita a cambios en la estructura de la producción vía el comercio exterior.

Semejante escepticismo sobre el alcance de las opciones tecnológicas se relaciona desde luego con la renuencia todavía considerable a abandonar la política de sustitución de importaciones en gran parte del mundo subdesarrollado. A pesar de la demostración del mundo real sobre lo que puede de hecho lograrse, permanecen obstáculos formidables al desmantelamiento del régimen de sustitución de importaciones. Los controles directos implican para la administración pública poder absoluto, así como ingresos suplementarios, que está poco dispuesta a entregar fácilmente. Más aún, el papel inevitablemente mayor de la empresa privada bajo cualquier régimen liberalizado recuerda la asociación con el colonialismo y da lugar a temores de entregas antisociales. Pero uno de los argumentos más poderosos de los políticos conservadores sigue siendo la supuesta rigidez de las opciones realmente disponibles. En gran medida esto resulta de deducir que las proporciones fijas son inevitables dada su existencia histórica durante la fase de sustitución de las importaciones. Nos proponemos investigar este tema, tratando primero de aclarar la naturaleza diferencial del proceso de innovación en cada fase, y demostrar luego el alcance potencialmente amplio de las innovaciones que emplean mano de obra, haciendo referencia a los casos del Japón histórico y a Corea y Taiwán contemporáneos.

\section{III}

Primero y ante todo, debe recordarse que a diferencia de un país industrializado donde el cambio tecnológico se ve como algo más bien automático y rutinario, o capaz de generarse mediante gastos en investigación y desarrollo experimental (IDE) de acuerdo con algunas reglas de análisis de costo-beneficio, en las sociedades contemporáneas en vía de desarrollo el cambio tecnológico no puede tomarse ni como automático ni como resultante de tales gastos en IDE. Ante esta situación' no podemos eludir la cuestión de cuál es el camino por el cual una economía subdesarrollada típica logra de hecho las innovaciones, dada la existencia de una gama de opciones tecnológicas en el exterior. Esta cuestión nos lleva a su vez a investigar más cuidadosamente las dimensiones siguientes: 1) la naturaleza precisa de la gama de tecnologías disponibles, 2) la disponibilidad de capacidad empresarial y gerencial inicial dentro del PVD, y 3) la naturaleza cambiante de la capacidad gerencial y empresarial requerida en el curso de la transición hacia el crecimiento moderno.

La gama de tecnologías desarrollada en las economías industriales maduras puede describirse mediante un conjunto de actividades unitarias que siguen una curva envolvente suave, tal como muestra la grafica 1. Una tecnología específica puede describirse como una curva en forma de $L$ que produce una unidad de producción con una pareja dada de coeficientes de capital y trabajo. La disponibilidad de tecnologías se compone del conjunto completo de actividades o tecnologías que se han encontrado factibles en algún país avanzado, en algún punto de la histo- 
ria, incluyendo el presente. Como existen varios países que exportan tecnología, por ejemplo los Estados Unidos, Alemania, el Reino Unido, Japón, con transferencias tecnológicas continuas entre ellos y con los PVD, es razonable postular la existencia de un solo conjunto tecnológico en todo el mundo que lo produce. Por ejemplo, la tecnología unitaria $A_{\mathrm{o}}$ pudo haber sido generada en Alemania en 1920, la $A_{1}$ en los Estados Unidos en 1920 , la $A_{2}$ en los Estados Unidos en 1950, etc. En otras palabras, a medida que nos movemos a la izquierda a lo largo de la gama tecnológica, llegamos a una tecnología más moderna, esto es, a una tecnología más reciente y de uso más intensivo de capital. A medida que aumenta el capital per capita esto significa que el obrero típico ha aprendido a cooperar con más unidades de capital de complejidad técnica creciente. En otras palabras, este proceso de profundización del capital es más complicado que la versión que da el libro de texto de trabajo "homogéneo" equipado con más unidades de capital "homogéneo".

\section{Gráfica 1}

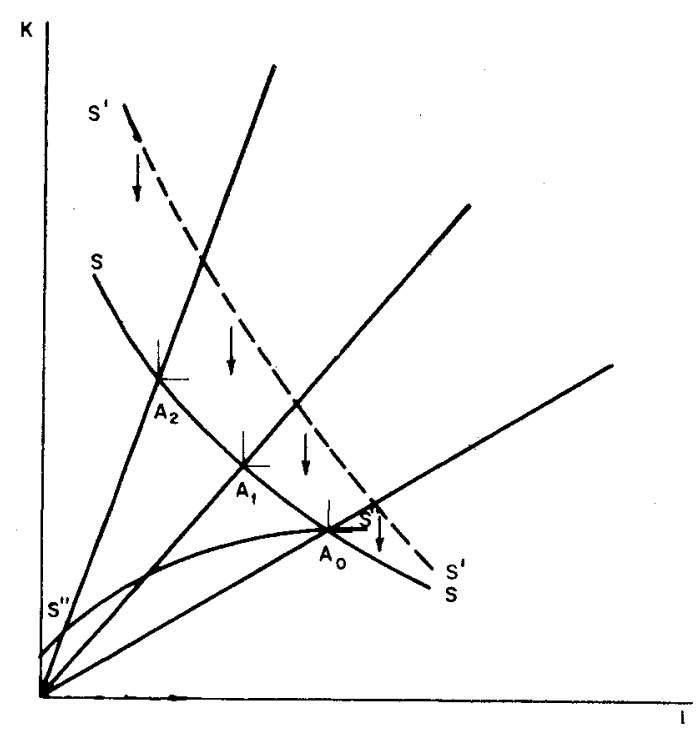

En cualquier punto del tiempo, un PVD típico está entonces en libertad de obtener una actividad unitaria específica de cualquier parte de este conjunto. La tecnología que se seleccione y lo que suceda como consecuencia última e inmediata de esa selección, es decir, los procesos y reacciones secundarios que se establezcan son, desde luego, parte del proceso innovador tomado en su conjunto. La calidad de ese proceso, cada paso de la trayectoria, dependen a su vez de la naturaleza de la capacidad empresarial, gerencial y de calificación laboral del que toma en uso la tecnología.

En consecuencia, el papel de la innovación debe verse como algo intimamente relacionado con la etapa en la que se encuentra la economía en desarrollo. En otras palabras, el papel del cambio tecnológico 
en la generación de producto y de empleo debe verse como algo sensible a las mismas fases distinguibles del crecimiento, a medida que la economía en transición va del agrarismo abierto al crecimiento económico moderno de Kuznets. En la primera fase postindependiente mencionada antes o de sustitución de importaciones, se hace un esfuerzo por aumentar la capacidad empresarial y el aprendizaje económico internos, en parte a través de la importación de personal técnico con los programas de ayuda, pero principalmente del sistema de protección establecido en las políticas del gobierno. De hecho, la explicación más razonable de la sustitución de importaciones es que ésta responde a una escasez real o imaginaria de espíritu empresarial y que permite ganar tiempo para desarrollar esta capacidad empresarial ${ }^{2}$ a través del aprendizaje en el trabajo o de procesos educativos más formales.

En términos de nuestra gráfica 1, esto significa que, aunque el conjunto de disponibilidad tecnológica pueda verse como indicado por la curva $S S$, las elecciones realmente disponibles al PVD durante la fase de sustitución de importaciones quedan mejor descritas por $S^{\prime} S^{\prime}$. En otras palabras, debido al estado inadecuado de la capacidad empresarial durante el principio del período de postindependencia de controles físicos, la eficiencia de generar producción por unidad de capital, en el país que recibe la tecnología, probablemente será sustancialmente menor que en el país que la da. Esto es probablemente más cierto cuanto más intensiva en el uso de capital sea la importación, es decir, mientras más alejamiento haya de la herencia cultural y de la experiencia del país receptor. Tales importaciones tecnológicas van con frecuencia acompañadas por ingenieros e inclusive administradores y supervisores importados -lo cual contribuye a los llamados proyectos completos de plantas industriales. Aún en la economía más atrasada es, desde luego, posible hacer operar en un sentido físico la tecnología más avanzada y refinada. Pero una nueva planta reluciente, puesta en una sociedad a muchos lustros de distancia, está condenada a ser en esencia menos eficiente. Esto es cierto por mil razones directas, tales como la ausencia de fuerza de trabajo con un mínimo de calificación, las posibilidades de subcontratación y reparación y mantenimiento internos, así como por muchas razones sociológicas sutiles que intervienen en el medio en el cual se pide que la planta opere. En otras palabras, la ineficiencia relativa será mayor cuanto más refinado y distante del resto de la economía sea el trasplante tecnológico, según indica la forma de la curva $S^{\prime} S^{\prime}$.

Si la economía se aleja de la fase de sustitución de importaciones, y entra en la segunda fase de liberalización y sustitución de exportaciones, probablemente haga aparición un segundo tipo de innovación, aun cuando no intencionado, que es una reducción en el grado de ineficiencia de la tecnología trasplantada originalmente. Llámese si se quiere eficiencia $X$, pero el costo del mero trasplante probablemente se reducirá, aun sin intención, es decir, en gran medida como resultado de factores externos

2 Unos cuantos países, como Malasia, que dominan una base de recursos naturales muy fuerte y confiable, pueden evitar por completo tal fase. Más aún, existen claramente conjuntos de sustitución de importaciones mejores y peores (esto es, más y menos costosos) de donde elegir, por ejemplo al comparar Brasil y Ghana, pero en el trabajo presente no podemos ampliar el examen de este tema tan interesante. 
a la conducta maximizante de la unidad productiva misma. Este aumento de la eficiencia productiva en el tiempo aumentará su importancia cuantitativa a medida que la artificialidad de la sustitución de importaciones se reduzca y surja una economía más competitiva. En la gráfica 1 los efectos del reforzamiento gradual de la eficiencia pueden representarse mediante las flechas que tienden en el tiempo a mover $S^{\prime} S^{\prime}$ hacia su posición original $S S .^{3}$

Otro tipo más consciente y cuantitativamente más importante de innovación comienza a adquirir importancia durante esta segunda fase de transición. Este fenómeno puede denominarse de asimilación de las innovaciones, es decir, de innovación de la tecnología importada en el sentido de utilizar relativamente más fuerza de trabajo abundante no calificada. A medida que la economía se desplaza de un patrón de crecimiento basado en los recursos naturales en la fase de sustitución de importaciones, a un sistema basado en los recursos humanos en la fase de sustitución de exportaciones, esto significa una sensibilidad creciente a la dotación de factores continuamente en cambio, primero en términos de la utilización eficiente de la fuerza de trabajo interna no calificada, y luego en términos de la incorporación del ingenio y calificación internos crecientes. En otras palabras, el tipo adecuado de tecnología finalmente aplicado debe ser aquel en el cual jueguen un papel importante no solamente la elección inicial de la tecnología disponible, sino también las adaptaciones y los ajustes hechos conscientemente a partir de ese momento como respuesta a cambios en los recursos internos y en las restricciones a la capacidad.

Mientras más liberalizada esté la economía en el sentido de la acción del gobierno como catalizador a través del mercado, por medios indirectos, más que como determinante directo del uso de los recursos a través de controles directos, mejores serán las posibilidades de que los millones dispersos de personas que toman decisiones puedan ser inducidos, por la pura fuerza de la maximización de utilidades, a tomar las decisiones "correctas". Aun en ausencia de cambio tecnológico, mientras la mano de obra excedente pese sobre el mercado -y las expectativas son que esta situación continúe en el futuro aún más intensamente- no podemos esperar más que un pequeño crecimiento de los salarios reales y poca capitalización. Sobre esto se sobrepone la conducta innovativa de asimilación mencionada la cual tiende, por la misma razón, a inclinarse a favor del uso de mano de obra. En la economía típica con abundancia de mano de obra -o con probabilidades de tenerla en el decenio siguiente (como es sin duda el caso de gran parte de África) - todo esto significa un ajuste tan eficiente de los servicios puros de fuerza de trabajo como sea posible. ${ }^{4}$ El que esto conduzca o no a un desplazamiento de la producción a favor de bienes de exportación intensivos en mano de

3 Es posible hacer un análisis más refinado, que establece diferencias entre la naturaleza ahorradora de capital y de trabajo de este movimiento, según la región donde opere la economía, pero no se introducirá aquí. Véase también "LDC Innovation Analysis and the Technology Gap" (con J. C. H. Fei), documento presentado ante la conferencia de la Asociación Económica Internacional sobre "La brecha entre las naciones ricas y las pobres" (The Gap Between Rich and Poor Nations, Nueva York, St. Martin's Press, 1972).

4 Es importante hacer hincapié en la palabra "eficiente" ya que no nos interesa aquí el objetivo, posiblemente también legítimo, de la creación de empleo como meta social por separado, como alternativa al aumento de la producción. 
obra, o a una combinación de productos dirigida predominantemente al mercado interno depende, por supuesto, ceteris paribus, del tipo de economía - por ejemplo, el tamaño. No es válido hacer ninguna generalización acerca de la importancia relativa de los desplazamientos en la combinación de productos, contra cambios en la tecnología con combinaciones dadas. Sin embargo, debe quedar claro que el asunto importante es que la búsqueda de innovaciones puede considerarse ahora como una actividad consciente del empresario individual y -dada la combinación de precios relativos más cercanos a la realidad, después de la liberalización, más una mayor capacidad empresarial- es probable que se dirija a la utilización de diversas formas de capital local colocado sobre la tecnología importada. Tal alargamiento del capital puede estar representado por una reducción en la relación capital/producto. El conjunto de tecnologías unitarias de asimilación posterior efectiva, esto es, después de la asimilación interna, puede así estar representado por la curva $S^{\prime \prime} S^{\prime \prime}$ de la gráfica 1 , donde la fuerza del esfuerzo local innovador intensivo en trabajo, está indicada por el desplazamiento "hacia abajo" del coeficiente de capital.

Debemos recalcar aquí que un conjunto tecnológico con pendiente negativa, por ejemplo $S S$, que representa el trasplante tecnológico puro, permite, a medida que se traslada a la izquierda, mayores niveles de productividad del trabajo, pero solamente a un costo creciente del capital. En un país caracterizado por la escasez de capital esto puede implicar desempleo técnico (a la Eckaus) y por lo tanto un menor ingreso per capita para la economía - a pesar del mayor nivel alcanzado de productividad de la mano de obra. Sin embargo, el "alargamiento" del capital interno puede afectar materialmente la situación al permitir el empleo de más mano de obra por unidad de capital. Si el conjunto de tecnología unitaria después de la asimilación, por ejemplo, $S^{\prime \prime} S^{\prime \prime}$, tiene pendiente positiva, a medida que la economía se traslada hacia la izquierda primero recibiendo del exterior y luego haciendo innovaciones internas, por encima de la tecnología obtenida, los niveles de productividad del trabajo mayores se vuelven congruentes con relaciones capital/productos menores. ${ }^{5}$

En resumen, una vez que el cuadro global de política económica se ha vuelto más favorable y ha permitido a la economía entrar en la segunda fase de transición, es esta capacidad local para "alargar" el capital lo que consideramos de la mayor importancia -en especial en las economías contemporáneas que se enfrentan a la formidable explosión de fuerza de trabajo pronosticada para los años setenta y ochenta. Es también en esta área específica donde es generalmente más pronunciado el escepticismo de los planificadores, ingenieros y funcionarios de los programas de ayuda, en especial con respecto a la gama de opciones tecnológicas realmente disponibles a la postre. Los ejemplos históricos del caso japonés, así como la evidencia contemporánea de Corea y Taiwán, nos permiten demostrar la existencia y la importancia potencial de tales innovaciones que "alargan" el capital en los PVD que posean fuerza de trabajo abundante.

5 La evidencia histórica dentro de los países desarrollados parece indicar una aproximación a la constancia de la relación capital/producto, esto es, $S^{\prime \prime} S^{\prime \prime}$, se aproxima a una posición horizontal que es el lugar geométrico "fínal" de las tecnologías unitarias. 
Como ha sido señalado por muchos observadores, incluyendo Allen y Lockwood, ${ }^{6}$ el rasgo más significativo del panorama japonés a principios del período Meiji — que siguió a dos siglos de aislamiento autoimpuesto, casi total- fue la capacidad de este país para elegir con relativa libertad de entre los elementos de un conjunto tecnológico perfeccionado en Occidente. La reapertura del comercio exterior y la reanudación de otros contactos, en especial el flujo de personal técnico en ambas direcciones, condujo de inmediato al estímulo del cambio tecnológico mediante la obtención directa. Pero mientras que los japoneses se han caracterizado a menudo por la posesión de una capacidad consumada para copiar e imitar, vale la pena hacer notar que, de hecho, muy pronto la mayor parte de las actividades innovativas internas "consistieron en la adaptación de técnicas extranjeras a las condiciones internas".?

Las razones por las que ocurrió relativamente temprano esta respuesta de la tecnología del sector industrial a las condiciones de dotación interna de recursos son complicadas y no se examinarán en este trabajo. Baste decir que en el período de posrestauración el Japón no adoptó políticas muy extensas o prolongadas de sustitución de importaciones -en parte debido a que la extraterritorialidad privó a este país de la capacidad para establecer aranceles protectores elevados a las importaciones, y en parte debido a que el gobierno consideró muy pronto que era más eficiente trabajar a través del mercado, es decir, mediante impuestos y subsidios, en lugar de con controles extensivos y propiedad pública de los medios de producción. Las fábricas del gobierno establecidas durante el período de restauración fueron consideradas principalmente como proyectos piloto y vendidas a la iniciativa privada en 1890. De este modo, el Japón se movió con relativa rapidez hacia la fase de sustitución de exportaciones.

Al investigar la importancia de las innovaciones que "alargan" el capital, es útil reconocer diferencias entre innovaciones relacionadas con la máquina propiamente dicha; aquellas que se refieren al proceso de producción general, que destacan la importancia de las actividades dentro de la planta, pero que son periféricas a la máquina; y las innovaciones con respecto al proceso de producción global, que tienen que ver con el tamaño y la organización de la planta en varias etapas del proceso.

Con respecto a las innovaciones que alargan el capital relacionadas con la maquinaria, el ejemplo más simple y, desde el punto de vista cuantitativo, probablemente el más importante fue la utilización de maquinaria inglesa y norteamericana a tasas y velocidades sustancialmente por encima de las utilizadas en el exterior. Por ejemplo, una vez que las lámparas de petróleo hicieron posible trabajar de noche, el tejido pudo ser hecho en dos

${ }^{6}$ George C. Allen, Japanese Industrialization: Its Recent Development and Present Conditions, Nueva York, Instituto de Relaciones del Pacífico, 1940; W. W. Lockwood, Economic Development of Japan, 1868-1928, Princeton, Nueva Jersey, Princeton University Press, 1954.

${ }_{7}$ M. Miyamoto. Y. Sakudo y Y. Yasuba, "Economic Development in Pre-Industrial Japan: 1859-1894", Journal of Economic History, diciembre 1965, p. 557. Los mismos autores informan también (p. 563) que durante el mismo período se hicieron innovaciones semejantes del capital o la tierra en el sector agrícola, principalmente a través de nuevos métodos de cultivo intensivos. 
turnos diarios, y a veces tres, con sólo dos o tres días de descanso al mes. Esto significa que la semana hábil promedio por máquina fue de dos $o$ tres veces la usual en el país de origen; y como la depreciación física es mucho menos importante que la obsolescencia económica, el uso de una máquina a dos veces su intensidad no la deteriora el doble. Este uso pesado de la maquinaria, típico del sector industrial japonés durante el siglo XIX, significó que la brecha normal entre la vida física y la económica de una máquina fue reducida de modo sustancial, y el capital fue "alargado" de manera considerable.

Por añadidura, se observó una aceleración del equipo de hilatura. Al trabajar la maquinaria a velocidades mayores o al sustituir por materias primas más baratas, por ejemplo: algodón crudo $-\mathrm{y}$ compensar esto aumentando el número de mujeres que manejaran el aumento resultante en el número de hilos rotos- se logró un ahorro sustancial de capital:

Deben hacerse notar ciertas diferencias importantes en las industrias de los dos países. La materia prima es esencialmente diferente. Aunque los japoneses usan cierta cantidad de algodón crudo norteamericano, la mayor parte de su algodón proviene de la India y es de fibra más corta, más susceptible de romperse -y requiere más trabajo para colocarlo en la máquina. La hilaza resulta con mayor proporción de fibra gruesa que requiere más mano de obra. Al añadir más trabajo a la operación, la máquina trabaja algo más que en la práctica norteamericana... Todos estos factores se relacionan de alguna manera a la política de mano de obra barata. Existen porque el trabajo es barato. ${ }^{8}$

Los husos japoneses estaban equipados con un rodillo frontal de $7 / 8$ de pulgada en lugar de uno de una pulgada, de modo de aceptar el algodón de fibra más corta al operar a velocidades mayores.

Por estas razones, es decir, las diferencias en el grueso de la hilaza y en la velocidad de la máquina, así como diferencias en el número de turnos, encontramos en la industria de los hilados que hubo una sustitución muy marcada entre capital y trabajo. Por ejemplo, Orchard informa que una hilandera japonesa competente que trabajara en una máquina de hilar del 20 , atendía de 300 a 400 husos, mientras que una hilandera norteamericana atendía de 1020 a 2688 husos, es decir, de $21 / 2$ a casi 7 veces. ${ }^{9}$ Tal como informa la Comisión de Aranceles de los Estados Unidos:

Para distribuir los costos fijos en la forma de costos de interés y depreciación elevados y percibir el mayor monto necesario para pagar dividendos a la tasa normal, se ha hecho el mayor esfuerzo por obtener la mayor producción posible del costoso equipo. La maquinaria es por lo tanto operada a una velocidad alta y, casi desde su inicio, las fábricas japonesas de hilados han estado operando noche y dia, empleando dos turnos de 12 horas (22 horas efectivas de trabajo) en un promedio de 27 días al mes. ${ }^{10}$

8 John C. Orchard, Japan's Economic Position, Nueva York, McGraw-Hill Book Co., 1930, p. 367.

9 Ibid., p. 367.

10 The Japanese Cotton Industry and Trade, Comision de Aranceles de los Estados Unidos, Government Printing Office, Washington, 1921, p. 99. 
Aquí, una vez más, dado un número estándar del hilo, las hilanderas japonesas atienden $\mathbf{2 4 0}$ husos, mientras que su contraparte norteamericano atiende alrededor de 1000 husos en la misma máquina. Todavía en 1932 el número de horas-hombre semanales por cada 1000 husos de la misma calidad fue de 328.8 en Japón contra 164.8 en Gran Bretaña y 143.1 en los Estados Unidos. ${ }^{11}$

Respecto del tejido de algodón puede hacerse una historia semejante. Una vez más:

...el elevado costo de la construcción de la planta se reduce considerablemente si se consideran las horas durante las cuales la planta se somete a un uso efectivo. Hasta ahora, las ruedas han girado durante 20 de cada 24 horas, mientras que en Europa se trabaja solamente 8 horas. El trabajo efectivo en Inglaterra es de menos de 38 horas a la semana, ya que 2 de éstas se dedican a la limpieza; en el Japón ésta se hace después de las horas de trabajo.12

De nuevo, la Comisión de Aranceles de los Estados Unidos informa que "en el tejido de lienzos de algodón, el tejedor japonés rara vez opera más de dos telares ordinarios, mientras que el tejedor norteamericano, quizá con ayuda en el suministro de bobinas nuevas, normalmente atiende de 8 a 10 telares del mismo tipo". ${ }^{13}$

Tal vez la evidencia más convincente de que estos ajustes en la máquina misma constituyeron una respuesta racional a diferencias muy marcadas en las dotaciones de factores fue que en el tejido, a diferencia del hilado, no se importó siempre el equipo automático extranjero más moderno. En vez de esto, muy frecuentemente se utilizaron los telares no automáticos disponibles, lo cual permitió un alargamiento mayor del que hubiera sido posible en el caso de tener que recurrir inicialmente a tecnologías más intensivas de capital. A diferencia de los países subdesarrollados de nuestros días, claramente el Japón no quiso importar por encima de su capacidad empresarial y de la habilidad de su mano de obra. ${ }^{14}$ Tal como explica la Comisión de Aranceles:

...el precio del telar automático es más del doble del telar ordinario, el cual, con el gasto adicional involucrado en la importación de los Estados Unidos o de Inglaterra, origina que el gasto total sea demasiado elevado en un país donde las tasas de interés bancarias son relativamente mucho mayores que el costo del trabajo. Por ello los gerentes de fábricas de tejidos japoneses han preferido hasta ahora emplear más trabajadores y no usar maquinaria más cara y ahorradora de trabajo,

11 The World Textile Industry: Economic and Social Problems, vol. 1, Oficina Internacional del Trabajo, Ginebra, 1937, p. 209.

12 Arnold S. Pearse, Japan and China, Informe de la Industria del Algodón, Federación Internacional de la Asociación de Hilanderos y Fabricantes Maestros de Algodón, Manchester, 1929, p. 86.

${ }_{13}$ The Japanese Cotton Industry and Trade, op. cit., p. 100.

14 La Comisión de Aranceles de los Estados Unidos, op. cit., p. 116, informa que un embarque de telares automáticos, importado poco después de principios del siglo se encontró tan difícil de operar, que después de quitarles las baterías y los movimientos de control, se trabajaron como telares ordinarios, con dos telares por cada tejedor. 
en contraste con la situación en los Estados Unidos donde el elevado precio de la mano de obra hace economizar en ésta y no en la máquina. ${ }^{15}$

Considerando en conjunto el hilado y el tejido simple de algodón en productos semejantes, la comisión concluyó que:

El hilador japonés promedio, o la tejedora media, atienden alrededor de una cuarta parte del número de husos o telares asignados que suele asignarse a una persona en una planta norteamericana. La compaparación del número total de personas empleadas en cada uno de los dos países para operar plantas individuales de tamaño semejante y, desde un punto de vista más amplio, la comparación del número total de personas empleadas en toda la industria norteamericana por cada 1000 usos, con el número que se requeriría sobre la misma base bajo las condiciones japonesas, confirma la relación general observada de que las fábricas japonesas requieren de tres y medio a cuatro veces más operarios que las norteamericanas. ${ }^{16}$

En el caso de la producción de seda, la cual, junto con el algodón, constituyó más del $70 \%$ de la producción industrial total hasta principios de siglo, se tienen evidencias semejantes sobre la capacidad de innovación sobre la máquina misma para alargar el capital. En la seda cruda, por ejemplo, los japoneses emplearon más del doble de mujeres que en Italia. En otras áreas, ya muy entrado el siglo xx, los ferrocarriles japoneses emplearon 19 trabajadores por milla de vía en comparación con 7 en los Estados Unidos. ${ }^{17}$ Acerca de la producción de artículos estampados, resulta instructivo el episodio siguiente:

Recientemente, un fabricante japonés de linóleos comunes decidió dedicarse a la producción de artículos estampados. Envió un representante a los Estados Unidos a comprar el equipo necesario. El representante estaba familiarizado con las máquinas modernas de estampado de linóleos, que imprimían varios colores a la vez y que producían hasta 15000 yardas cuadradas en 9 horas, pero consideró que el equipo era demasiado caro, en especial ya que su mano de obra ganaba sólo 50 centavos de dólar por día, así que buscó un viejo equipo de impresión manual en una planta norteamericana. No estaba en venta. Sus piezas estaban dispersas en un viejo almacén de la fábrica. Algunas de ellas tenían 40 años y todo el equipo había sido desechado 15 años antes. Pero el representante japonés lo compró y lo embarcó al Japón. En el pago inmediato ahorró dinero dado que compró el equipo al precio de solamente una máquina de estampado o inclusive a menos del precio de un equipo nuevo, pero instaló en su planta equipo que en Estados Unidos sólo se destinaba a desecho. En el Japón inició una nueva industria en una etapa de desarrollo técnico que se había vuelto obsoleta años antes en los países industriales más viejos. ${ }^{18}$

15 The Japanese Cotton Industry and Trade, op. cit., p. 116. Un ejemplo semejante de la flexibilidad técnica más allá de lo que la mayoría de los ingenieros admitirían es proporcionado por el telar automático Toyoda, uno de los pocos inventos japoneses en este campo. Fabricado después por Platt y Oldham bajo una patente japonesa, en Inglaterra se anunció que requería 20 mujeres por telar; en el Japón se habían utilizado siempre 50 mujeres.

16 Ibid., p. 113.

17 Orchard, op. cit., p. 375.

18 Ibid., p. 246. 
Muchos de los trabajadores adicionales de las plantas japonesas no estaban dedicados a la máquina en sí, sino a lo que podrían llamarse actividades periféricas o de manejo manual. En lugar de bandas mecá. nicas se idearon cadenas humanas. El empacado se hacía con mayor frecuencia a mano. Como informa Orchard también, "en una de las mayores fundidoras de cobre del Japón, la arcilla para el recubrimiento de los hornos era acarreada en la espalda por mujeres desde una colina cercana. En la planta de la Compañía de Gas Tokyo, el coque se ponía en sacos manualmente y luego éstos eran acarreados por coolies, algunos de ellos mujeres, a las barcazas de los canales adyacentes. El carbón, aun en las plantas más grandes de Tokio, se descarga a mano y es acarreado en canastos hacia las generadoras. ${ }^{19}$ La capacidad para sustituir capital por trabajo en tales actividades periféricas al parecer existió y su incidencia era considerable. Con mucha frecuencia tales actividades eran marcadas por el ritmo de la máquina en el sentido de Hirschman, esto es, aunque pudieran parecer un desperdicio para el ojo occidental, eran de hecho llevadas al ritmo de máquinas bien espaciadas como parte de la misma línea de producción que contenía gran cantidad de trabajadores no calificados. ${ }^{20}$

Un tercer tipo de innovación que alarga el capital, del cual se ha hecho gran uso en la historia del Japón, es la variedad que podría llamarse de "ahorro de planta". Este tipo se caracteriza a menudo por la coexistencia de diferentes etapas históricas de producción en la misma industria. La producción de seda cruda y la de tejidos de algodón son ejemplos notables. En la primera de estas industrias la cría de gusanos y la producción de capullos eran manejadas principalmente por las esposas de agricultores en crías caseras pequeñas, como extensiones de la finca rural. En el tejido de algodón, la mayor parte de la hilaza se daba a maquila en los hogares agrícolas, con telares individuales dispersos en granjas y talleres. Pero inclusive en las fábricas de hilados modernas, los procesos preparatorios y de acabado se realizaban en gran medida en pequeñas unidades.

Esta superviviencia más bien notable de la industria interna en la forma de maquila debe explicarse en gran medida en términos de la explotación de la complementariedad entre muchas unidades pequeñas intensivas de trabajo y la gran unidad industrial. El intermediario tradicional, como representante de la unidad de subcontratación, servía tanto como abastecedor y como mercado para los bienes elaborados domésticamente. Se desarrolló una especialización de funciones entre los talleres, aun entre los miembros de una familia. De este modo pudieron lograrse economías bajo un solo techo, es decir, usando a nivel de taller, trabajo barato en colaboración con maquinaria anticuada mientras que podrían lograrse economías de escala considerables en el financiamiento, la compra y la venta de las mercancias. ${ }^{21}$ Es muy notable la importancia relativa con-

19 Ibid., p. 255.

20 Esto es muy semejante a los métodos contemporáneos de construcción mediante el uso de concreto reforzado en la India y Pakistán. Aquí las mezcladoras de cemento se encadenan al vaciado final del concreto mediante una larga fila de trabajadores que se pasan el cemento de mano en mano; el cemento se coloca justo antes de estar listo para enfriarse y fraguar.

21 "En ocasiones inclusive una pieza simple no se completa en un taller $u$ 
tinuada de esta empresa de tipo casero; la industria casera contribuyó con más de dos terceras partes del producto industrial en 1878, casi $60 \%$ en 1895, y retuvo importancia considerable ya muy entrado el siglo xx. No solamente la producción de objetos de laca, porcelana, alfarería, sake, enlatado de pescado y frutas, sino también la de bienes de consumo nuevos que surgieron posteriormente tales como bicicletas, lámparas y productos de caucho, habrían de mostrar las mismas características.

De la inversión en plantas y equipo, en la mayoría de los países la primera representa más del $50 \%$ de la inversión total. La capacidad para utilizar hogares para llevar a cabo operaciones de maquila, y de este modo reducir los gastos en planta, representó sin duda un tipo importante de innovación "alargadora" del capital. Al ser desplegado entre las familias, pero mejorando la maquinaria de un gran número de plantas pequeñas dispersas, grandes cantidades de trabajo no calificado podían utilizarse tanto en la producción directa como en la satisfacción del aumento resultante de la demanda de actividades de transporte y manejo de los productos. De este modo, los empresarios japoneses pudieron primero incorporar servicios de mano de obra puros, y después el ingenio y la calificación domésticos, a los procesos industriales, en gran medida de exportación.

Un examen de la relación capital/trabajo en el sector no agrícola japonés durante el período examinado indica la efectividad de las innovaciones que alargan el capital a nivel agregativo, es decir, mientras que la tasa anual media de intensificación del uso de capital fue de $4.3 \%$ entre 1906 y 1917, el período anterior, de 1892 a 1900, se caracterizó por una tasa de $2.8 \%$ anual, que disminuyó al $1.7 \%$ entre 1900 y 1906 .

Esto parece sugerir al menos que los empresarios mejoraban a través de un proceso de aprendizaje en el trabajo, mediante innovaciones orientadas al uso mayor de mano de obra, antes de que la situación de mano de obra abundante desapareciera, una vez que el ejército de reserva de desempleados y subempleados quedó absorbido sustancialmente después de la primera guerra mundial.

En Corea, la devaluación en 1964 y una reforma sustancial en la tasa de interés en 1965 sentó la base de los cambios importantes tecnológicos y de composición del producto. En la industria textil, electrónica y de contrachapado de madera abundan los ejemplos de adaptaciones de tecnología importada que alargan el uso del capital. En las manufacturas de seda, por ejemplo, una mujer opera dos telares contra 6.8 telares en el Japón de hoy. Como reacción al alza actual de los salarios en el Japón, Corea está acaparando la venta de hilo de seda de baja calidad en el cual pueden emplearse más mujeres para compensar la calidad inferior de la materia prima. En el tejido de algodón, una mujer coreana maneja tres telares, contra cuatro en el Japón; en los hilados el contraste es de 600 y 900 husos. Más aún, la maquinaria coreana es operada en tres turnos de ocho horas diarias en contra de solamente dos en el Japón. En los trabajos periféricos a las máquinas, podemos notar que actualmente se hace en el Japón uso de bandas de proceso, por ejemplo, en 
las operaciones de cardado y peinado, mientras que en Corea el transporte de los materiales se hace manualmente.

En la producción de contrachapados de madera, lo que parece a primera vista un proceso de producción muy semejante al realizado en los Estados Unidos, o sea, de proporciones fijas, resulta ser de hecho muy flexible, lo cual es muy interesante debido principalmente a la mayor velocidad de la máquina, combinada con métodos de reparación mucho más intensivos en el uso de mano de obra. En los Estados Unidos, la máquina elimina en forma automática las piezas de madera defectuosas. En el Japón, las piezas defectuosas son cortadas a mano y la sección es desechada. En Corea, las secciones defectuosas son cortadas a mano, los desperdicios se ahorran, y los defectos son rellenados a mano. Aquí de nuevo la materia prima de menor calidad puede mejorarse a una calidad equivalente a través de la aplicación de mano de obra barata. En consecuencia, en Corea, encontramos el doble de trabajadores por unidad de capital, es decir, 123 trabajadores operan en una línea de producción en contraste con 72 en el Japón; más aún, una línea coreana es operada 22 horas al día en comparación con 20 en el Japón. Al mismo tiempo, en la inspección, reparación, mantenimiento de materiales en proceso y de la maquinaria se emplean de 10 a $15 \%$ más trabajadores.

En la industria electrónica, se encuentran las innovaciones más destacadas para utilizar más fuerza de trabajo con la máquina. En las operaciones de armado de transistores, por ejemplo, donde las tasas de salarios son 10 veces mayores que las que reciben operadores equivalentes en los Estados Unidos (en la misma empresa), la maquinaria es operada a toda su capacidad física, esto es, seis días, tres turnos al día, lo cual es $20 \%$ por encima del equivalente en Estados Unidos. Más aún, ciertas operaciones especiales tales como la alimentación y el empacado, de ordinario se hacen manualmente sobre la línea de ensamble, en lugar de hacerse automáticamente. A pesar del mayor uso de trabajo, la productividad por trabajador parece ser mayor debido en parte al proceso de aprendizaje más rápido (se afirma repetidamente que toma cuando menos dos semanas menos adiestrar en el armado a mujeres coreanas que a norteamericanas) pero principalmente a la mayor disciplina y atención en la línea de ensamble a todo lo largo. Por ejemplo, en una empresa (IMEC) la diferencia en la velocidad de ensamble en un equipo idéntico da una diferencia de $30 \%$ en la producción (de 68 unidades por hora-máquina a $85)$, y en un proceso de montaje de troqueles se eleva a más de $100 \%$ (de 113 unidades por hora a 240). Estas mayores velocidades de operación, ya sea debido al ritmo mayor de la máquina o del operador, van de nuevo acompañadas por el empleo de más mujeres en las etapas de prueba, inspección y reparación, que lo que se observa en el Japón o los Estados Unidos. Las piezas defectuosas no son eliminadas sino reparadas a mano. De igual manera, al trabajar la maquinaria a toda su capacidad, se dedica más fuerza de trabajo al mantenimiento y a la reparación del equipo de capital.

Respecto a otras opciones de organización y tecnológicas relacionadas con la planta, el fenómeno más importante reside claramente en el área de la subcontratación o maquila, tanto interna como internacionalmente. La subcontratación interna de fabricantes locales de equipo y refacciones se practica cada vez más, en especial en la industria electrónica; 
en ocasiones, tal como indica la experiencia de varias compañías, se requieren de dos a tres años para que el suministrador local, después de un proceso de aprendizaje en el trabajo, se convierta en un productor a costo más bajo que la planta matriz o las alternativas de importación. Tales innovaciones ahorradoras de capital, principalmente aquellas que se logran a través de la reducción en gran escala de los gastos fijos de la planta y urbanos, aumentan acentuadamente en varias otras industrias, aunque no se encuentran aún tan difundidas en Corea como en el Japón imperial (y en Taiwán contemporáneo). Internacionalmente, desde luego, la subcontratación de la fase intensiva de trabajo de un proceso de producción multietápico y que precisa tecnología, es una manera potencialmente muy eficaz para ocupar los servicios de trabajo virtualmente puros en el proceso de desarrollo. Los esquemas de procesamiento de exportaciones maquiladas, que consisten en zonas fiscales libres de impuesto en las cuales, a menudo bajo convenios subsidiarios o de subcontratación con las empresas japonesas o norteamericanas, las materias primas son importadas y luego reexportadas, después que se les ha añadido valor en la forma de trabajo barato, pueden ser de la mayor utilidad. Este esquema genera ahora alrededor del $20 \%$ del volumen de exportación de Corea, que en sí mismo se ha estado elevando a unas tasas medias anuales casi increíbles de entre 30 y $40 \%$ en los últimos tres años.

Pero es hacia la cuestión más importante de la absorción productiva de trabajo a través de cambios en la combinación de productos y el comercio que debemos volver la vista. A este respecto, en 1962 los alimentos y las materias primas producto de la tierra constituyeron en Corea el $75 \%$ de las exportaciones totales, mientras que la industria manufacturera ligera que incluye madera contrachapada, seda cruda, textiles de algodón, pelucas y calzado, representó globalmente el 15\%. Para 1968 la situación se había invertido por completo: el $77 \%$ de las exportaciones eran manufacturas y solamente el $14.5 \%$ alimentos, ganadería y materias primas. Debe hacerse notar además que las exportaciones industriales producidas en pequeña escala, es decir, en unidades de menos de 10 obreros que son sin duda la parte más intensiva de trabajo de la gama de actividades, crecieron de $18.6 \%$ del total en 1963 a 31.4 en 1968 .

Como consecuencia de todo esto, el empleo industrial se duplicó entre 1963 y 1969, en particular en la industria ligera, donde el empleo creció a una tasa superior inclusive a su valor agregado (véase el cuadro 1).$^{22} \mathrm{Al}$ menos hasta que Corea empezó a invertir su tendencia liberalizadora, después de $1968,{ }^{23}$ la relación capital/trabajo global en la industria descendió a partir de 1964 (véase la gráfica 2). Tenemos también evidencia, por ejemplo en el cuadro 2 , de que existe aquí una considerable disparidad por escala tal como en muchos otros países menos "bien comportados". ${ }^{24}$ Esto se debe a las bien conocidas discrepancias en las deformaciones residuales de los precios de los factores que afectan a

22 Agradezco la ayuda del profesor Sung-Hwan Jo de la Universidad Sogang, Seúl, en este contexto.

${ }_{23}$ Este cambio es parte de un problema más largo de contar y que no podemos tratar en este trabajo.

${ }_{24}$ De Paul Kuznets, Labour Absorption in Korea Since 1963, trabajo presentado a la Conferencia de Problemas de la Mano de Obra en el Este y el Sudeste de Asia, op. cit. 


\section{Cuadro 1}

CoREA: INCREMENTo dE LA FUERZA dE TRABAjo INDUSTRIAL, 1955-1967

\begin{tabular}{lcc}
\hline Año & $\begin{array}{c}\text { Empleo de la fueraa } \\
\text { de trabajo industrial } \\
\text { (miles) }\end{array}$ & $\begin{array}{c}\text { Tasas anual media de } \\
\text { incremento }\end{array}$ \\
\hline 1952 & & \\
1953 & & \\
1954 & 2223 & \\
1955 & 2716 & \\
1956 & 2444 & \\
1957 & 2729 & \\
1958 & 3062 & \\
1959 & 2139 & \\
1960 & 3136 & \\
1961 & $3.5 \%$ \\
1962 & 3360 & \\
1963 & 3809 & \\
1964 & 4199 & \\
1965 & 4312 & \\
1966 & 4680 & \\
1967 & & \\
1968 & & \\
1969 & & \\
\hline
\end{tabular}

empresas grandes y pequeñas, siendo en estas últimas los salarios reales más bajos y los costos de capital mayores.

La información microeconómica pertinente es difícil de obtener, ya que las entrevistas a empresas resultaron inútiles para obtener información confiable a nivel de empresa. Sin embargo, en la mayor compañía maderera coreana, se observó una disminución en su relación capital/ trabajo: de 35.8 en 1965-1966 a 27.8 en 1968-1969.

También en Taiwán, una vez que las políticas de liberalización puestas en marcha a principios de los años sesenta hubieron reducido sustancialmente algunas de las principales deformaciones de los precios relativos, comprendidas en la sustitución de importaciones, ocurrieron innovaciones muy intensivas de trabajo en las industrias textil, electrónica y de procesamiento de alimentos. La producción de hongos y espárragos

Gráfica 2

COREA: RELACión CAPITAL/TRABAjo

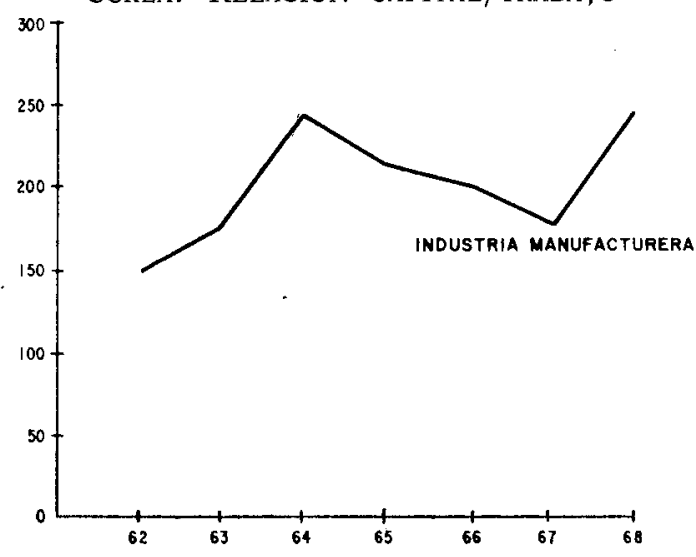




\section{Cuadro 2}

Corea: Densidad de capital POR TAMaño de ESTABlecimiento EN LAS MANUFACTURAS Y LA MINERÍA, 1968

\begin{tabular}{cccc}
\hline $\begin{array}{c}\text { Tamaño del } \\
\text { as tableoimiento }\end{array}$ & Aativos Fijos & $\begin{array}{c}\text { Nimero de } \\
\text { trabajadores }\end{array}$ & $\begin{array}{c}\text { Relación } \\
\text { Capital trabajo }\end{array}$ \\
\hline $5-9$ & 23772 & 82183 & .289 \\
$10-19$ & 28601 & 79141 & .361 \\
$20-49$ & 38367 & 100586 & .381 \\
$50-99$ & 28695 & 73467 & .399 \\
$100-199$ & 28406 & 76485 & .371 \\
$200-499$ & 68 & 120875 & .567 \\
$500 \mathrm{y}$ mas & 188421 & 258446 & .729 \\
\hline
\end{tabular}

Fuente: EPB-KDB-Report on Minning and Manufacturing Census, 1968 y Paul Kuznets, "Labour Absorption in Korea Since 1963", trabajo presentado en la Conferencia, sobre Poblemas de Mano de Obra del Este y del Sudeste de Asia, Singapur, mayo 22-28 de 1971, cuadro 8.

en gran escala, como forma de empleo agrícola accesorio (semejante al caso de la seda en el Japón) ${ }^{25}$ combinada con procesamientos relativos y actividades de enlatado, proporcionaron mercados importantes para el excedente de fuerza de trabajo no calificada, en especial la femenina. Al paso del tiempo, y facilitada por el establecimiento de la Zona Kaohsiung de Procesamiento de las Exportaciones, ocurrió la sustitución de exportaciones a través de una expansión fuerte de industrias intensivas en el uso de trabajo.

A nivel microeconómico, con base en una entrevista a 20 empresas, la mayor parte del alargamiento del capital ocurrió claramente en la fabricación, en contraste con los procesos continuos. ${ }^{26}$ Una gran fábrica de plásticos producía tanto materias primas (plásticos PVC) como productos terminados (varios productos de plástico incluyendo muebles y juguetes). El presidente de su junta directiva informó que en el proceso continuo (de producción de resina para los plásticos PVC) la relación capital/trabajo de su planta era aproximadamente la misma que la de otras de la compañía matriz en los Estados Unidos, pero que en el proceso de fabricación la relación capital/trabajo era alrededor de la mitad de la de las plantas estadounidenses.

En la industria de ensamble electrónico es donde se hace uso más intensivo del trabajo. Aunque en los Estados Unidos las piezas son armadas principalmente con la ayuda de máquinas, en Taiwán este trabajo es realizado por mujeres. De acuerdo con el gerente general de una de las principales empresas electrónicas, la cantidad de trabajo utilizada en el armado de un televisor en una planta de Taiwán es alrededor de $50 \%$ mayor que el equivalente en la planta matriz en los Estados Unidos. De hecho, la mayor parte de las empresas electrónicas entrevistadas estaban haciendo esfuerzos de una $u$ otra manera para introducir métodos intensivos en el uso de trabajo. No obstante que las relaciones capital/trabajo en esta industria generalmente han aumentado con el tiempo, la empresa electrónica más grande de Taiwán ha experimentado un aumento del ca-

25 De hecho, tales percepciones no agrícolas constituyeron en 1962 el 72\% de los ingresos agrícolas.

${ }^{2}$ Sobre este punto agradezco la ayuda del profesor N. R. Chen de la Universidad Cornell. 
pital de nueve veces y un aumento del empleo de 16 veces entre 1965 y 1969. En la industria electrónica, al parecer las relaciones capital/trabajo han descendido durante los años sesenta. Muchos de los gerentes entrevistados señalaron que la nómina era menor, a pesar del volumen sustancialmente mayor de empleo.

Una conclusión provisional que podría derivarse de estas visitas es que mientras más cercano esté el proceso de producción a la etapa de procesamiento de las materias primas, esto es, la integración hacia atrás, menores serán las posibilidades de lograr una sustitución eficiente de capital por trabajo, o de lograr dinámicamente innovaciones alargadoras del capital; mientras más cercano esté el proceso a la etapa de acabados, mayores serán tales posibilidades.

\section{Cuadro 3}

TAIWÁN: INCREMENTO DE LA FUERZA DE TRABAJO INDUSTRIAL, 1952-1969

\begin{tabular}{|c|c|c|}
\hline A מ̆o & $\begin{array}{c}\text { Empleo de la fuerza } \\
\text { de trabajo industrial } \\
\text { (miles) }\end{array}$ & $\begin{array}{l}\text { Tasa anual media de } \\
\text { incremento }\end{array}$ \\
\hline $\begin{array}{l}1952 \\
1953 \\
1954 \\
1955 \\
1956 \\
1957 \\
1958 \\
1959\end{array}$ & $\begin{array}{ll}1 & 198 \\
1 & 223 \\
1 & 261 \\
1 & 291 \\
1 & 304 \\
1 & 366 \\
1 & 418 \\
1 & 481\end{array}$ & $3.0 \%$ \\
\hline $\begin{array}{l}1960 \\
1961 \\
1962 \\
1963 \\
1964 \\
1965 \\
1966 \\
1967 \\
1968 \\
1969\end{array}$ & $\begin{array}{ll}1 & 536 \\
1 & 598 \\
1 & 689 \\
1 & 781 \\
1 & 843 \\
1 & 938 \\
2 & 034 \\
2 & 239 \\
2 & 480 \\
2 & 679\end{array}$ & $6.4 \%$ \\
\hline
\end{tabular}

En 1952 el arroz y el azúcar constituyeron el 78\% de los ingresos en Taiwán por concepto de exportación. Para 1969 esta participación se había reducido a $4.8 \%$. Durante el mismo período los productos agrícolas no tradicionales, incluyendo frutas y vegetales frescos y enlatados, se elevaron de cero a $10 \%$ del total, y lo que es más impresionante, los bienes manufacturados, incluyendo madera y productos contrachapados, se elevaron de 5 a $69 \%$ del total. Las dimensiones plenas de este cambio estructural se aprecian cuando de nuevo notamos que los ingresos totales por exportaciones se elevaban con gran rapidez, a tasas superiores a $20 \%$ anual, en especial durante los sesenta.

A nivel macroeconómico y consecuentemente con todo esto, el empleo industrial creció a tasas de $3.0 \%$ anual entre 1952 y 1959 (véase el cuadro 3), pero se aceleró al $8.1 \%$ anual en el decenio de los sesenta, una vez terminada la transición a la promoción de las exportaciones. Si dividimos el sector no agrícola en los sectores $M$ y $S$ de Kuznets, vemos que en el sector $M$ la tasa de absorción de empleo se elevó de $4.6 \%$ anual en $1950-1960$ a $7.5 \%$ en $1960-1969$, y de $3.2 \%$ a $6.5 \%$ en el sector $S .^{27}$

${ }_{27}$ Estos datos son de Harry Oshima, Experience of Labour Absorption in Fostwar Taiwan, ponencia presentada a la Conferencia Sobre Problemas de la Fuerza de Trabajo en el Este y el Sudeste de Asia, Singapur, mayo 22-28 de 1971. 
Es igualmente significativo el hecho de que una vez consolidadas las reformas a las tasas de interés y otras -además de las reformas al tipo de cambio y agrarias que se adoptaron con anterioridad- esta tendencia parece haberse acelerado, esto es, en el período 1964-1965 las tasas correspondientes son $8.7 \%$ en el caso del sector $M$ y de $7.2 \%$ en el caso de $S$.

Este enfoque anecdótico, aunque se admite que no constituye una prueba, es congruente con nuestro argumento principal de que un país en desarrollo, en especial uno abierto y de tamaño mediano - siempre que se produzcan los cambios adecuados de política económica-, puede esperar una transición de la sustitución de importaciones, con transplante tecnológico puro, a la sustitución de exportaciones, donde adquieren relevancia las innovaciones que utilizan más trabajo. Una vez corregidas las escaseces de capacidad empresarial y otros costos económicos - y reducido el ambiente de protección artificial- los cambios tecnológicos intensivos en el uso de trabajo, de tipo intencional o no, adquirieron importancia creciente. En esta fase el famoso conflicto entre los objetivos de producción y empleo en el desarrollo industrial pueden ponerse en duda. Tanto la experiencia histórica del Japón como la de Corea y Taiwán en años recientes ilustran que el escepticismo corriente de nuestros días en relación con la supuesta tiranía de los coeficientes técnicos rígidos puede estar seriamente errado. Este error se deriva principalmente de una subestimación de la inventiva potencial de los empresarios locales, una vez que tienen acceso de acuerdo con un cierto precio, a los insumos requeridos. Este no es un asunto inconsecuente. Porque en esto, si nuestro escepticismo no se justifica, constituiría uno de los argumentos más poderosos para acelerar la tendencia corriente, más bien lenta y dispareja, hacia la liberalización y la erosión de las diferencias entre los precios sombra o "reales" y los del mercado en los mercados de bienes y factores. 